\title{
Altered expression of microRNA-98 in IL-1 $\beta$-induced cartilage degradation and its role in chondrocyte apoptosis
}

\author{
JING WANG $^{1 *}$, LINGQING CHEN ${ }^{2 *}$, SONG JIN $^{1}$, JUN LIN $^{1}$, HONGMEI ZHENG ${ }^{1}$, \\ HONG ZHANG ${ }^{1}$, HONGTAO FAN ${ }^{1}$, FANG HE ${ }^{1}$, SHA MA $^{1}$ and QIN LI $^{1}$ \\ ${ }^{1}$ Department of Rheumatology and Immunology, First People's Hospital of Yunnan; ${ }^{2}$ Department of Orthopedics, \\ First Affiliated Hospital of Kunming Medical University, Kunming, Yunnan 650032, P.R. China
}

Received December 29, 2015; Accepted March 23, 2017

DOI: $10.3892 / \mathrm{mmr} .2017 .7028$

\begin{abstract}
Osteoarthritis (OA) is a multifactorial disease characterized by degeneration of the articular cartilage due to genetic and epigenetic components. The pathogenesis of $\mathrm{OA}$ is complex and the mechanism of chondrocyte homeostatic regulation remains to be fully elucidated. Previous studies have demonstrated that microRNAs (miRNAs/miR) contribute to cartilage dysfunction. However, the functional role of miR-98 in interleukin-1 $\beta$ (IL-1 $\beta$ )-induced chondrocyte apoptosis in OA cartilage remains to be investigated. The present study aimed to identify and characterize the expression profile of miR-98 and apoptosis-associated proteins in healthy and OA chondrocytes, and western blot analysis and TUNEL staining were used to evaluate the role of miR-98 in the regulation of chondrocyte apoptosis. The present study demonstrated that miR-98 expression was increased in OA chondrocytes in response to IL- $1 \beta$ stimulation, and the expression levels of apoptosis-associated proteins, including Fas cell surface death receptor, caspase-3, caspase- 8 and B-cell lymphoma-2 (Bcl-2)associated $X$ protein, were also increased in IL-1 $\beta$-stimulated chondrocytes. In addition, it was revealed that upregulation of miR-98 was accompanied by reduced expression of Bcl-2 following exposure to IL- $1 \beta$. IL-1 $\beta$-induced downregulation of Bcl-2 was associated with miR-98-mediated translational repression. Transfection of OA chondrocytes with a miR-98 inhibitor had an inhibitory effect on IL-1 $\beta$-induced cell apoptosis, increased cell proliferation and upregulated $\mathrm{Bcl}-2$ expression. It is possible that miR-98 inhibited IL-1 $\beta$-induced chondrocyte apoptosis by modulating Bcl-2 expression
\end{abstract}

Correspondence to: Professor Qin Li, Department of Rheumatology and Immunology, First People's Hospital of Yunnan, 157 Jinbi Street, Kunming, Yunnan 650032, P.R. China

E-mail: liqinkhyy@163.com

*Contributed equally

Key words: microRNA-98, osteoarthritis chondrocytes, B-cell lymphoma-2, apoptosis-associated proteins, interleukin-1 $\beta$ levels. The findings of the present study indicated that the effects of miR-98 on chondrocyte apoptosis were induced by regulation of Bcl-2 expression. In addition, the present study confirmed that miR-98 targeted the 3'-untranslated region of Bcl-2. In conclusion, miRNA-coordinated regulation of apoptosis-associated protein expression has been identified in OA chondrocytes following IL-1 $\beta$ induction.

\section{Introduction}

Osteoarthritis (OA) is a prevalent degenerative joint disease, which is characterized by progressive destruction of the articular cartilage and subchondral bone. Numerous etiological factors are implicated in the pathogenesis of OA; however, cartilage destruction appears to be due to chondrocyte apoptosis (1). It has previously been suggested that microRNAs (miRNAs/miR) are involved in the pathogenesis of OA (2). miRNAs are single-stranded, non-coding small RNAs that negatively regulate the expression of target genes in a post-transcriptional manner; $>700$ miRNAs have been identified to serve a role in the regulation of cellular processes, including proliferation, apoptosis and differentiation $(3,4)$. Previous studies that used miRNA microarrays to compare the expression of miRNAs between normal and OA human articular cartilage revealed that miRNAs were comprehensively involved in OA generation (5,6). Diaz-Prado et al (6) previously generated cartilage-specific Dicer-null mice, which exhibited reduced chondrocyte proliferation and severe growth defects, thus indicating the importance of miRNAs in regulating cartilage function. However, the expression and functional role of miRNAs in OA remains to be fully elucidated. Therefore, the miRNA expression pattern and functions that are essential for chondrocyte development and OA damage require further investigation. The inflammatory cytokine interleukin (IL)-1 $\beta$, which is a major catabolic inducer, is one of the most prominent catabolic cytokines, which serves a crucial role in OA pathogenesis $(7,8)$. IL-1 $\beta$ promotes the production of proteases, such as matrix metallo proteinases (MMPs), and inhibits the synthesis of proteoglycans and collagens by chondrocytes $(9,10)$. Previous studies have suggested that various miRNAs are involved in IL-1 $\beta$-induced OA cartilage destruction, including miR-9, miR-98 (11), miR-140 (12), miR-146a (13) and miR-558 (14). 
Previous studies have investigated the role of miR-98 in the regulation of tumor growth, invasion, angiogenesis and anti-inflammation in various disease models. Siragam et al (15) defined a regulatory role for miR-98 in tumor angiogenesis and invasion via the suppression of activin A receptor type $1 \mathrm{~B}$ and MMP11 expression. Du et al (16) suggested that miR-93, miR-98 and miR-197 exert a negative regulatory effect on the expression of tumor suppressor gene FUS1 in lung cancer. Li et al (17) demonstrated that the expression levels of miR-98 were reduced in melanoma tissues at a higher tumor stage and in melanoma with metastasis, and suggested that miR-98 inhibited melanoma metastasis via a novel miR-98-IL-6-negative feedback loop. However, the expression pattern and functional role of miR-98 in chondrocyte apoptosis of OA cartilage remains to be fully elucidated. The present study aimed to determine the molecular mechanism underlying the pathogenesis of OA. Therefore, the expression levels of miR-98 in normal chondrocytes and IL-1 $\beta$-treated OA chondrocytes were detected. In addition, the role of miR-98 in an IL-1 $\beta$-induced OA disease model was identified, suggesting its potential involvement in the regulation of apoptosis. The role of miR-98 in the regulation of chondrocyte proliferation and apoptosis was investigated using an in vitro OA model. The findings of the present study confirmed that miR-98 targeted the 3'-untranslated region (3'-UTR) of B-cell lymphoma 2 (Bcl-2). These findings suggested that miR-98 serves a crucial role in the coordinated regulation of the expression of apoptosis-associated proteins in chondrocytes in response to IL-1 $\beta$-induction.

\section{Materials and methods}

Mouse primary chondrocyte culture. The present study was approved by the Ethics Committee of the First People's Hospital of Yunnan Province (Kunming, China). A total of 30 2-week old male C57BL/6 mice ( $\sim 10 \mathrm{~g})$ were purchased from the Shanghai Animal Center, Chinese Academy of Sciences and housed in a specific pathogen free facility with a constant humidity and temperature at 12:12 h light:dark cycle with free access to food and water for 2 days in the animal research facility in accordance with an approved protocol. Mice were anesthetized with diethyl ether (60297; Sinopharm Chemical Reagent Co., Ltd., Shanghai, China) and sacrificed, then cartilage sections of mice were aseptically removed and washed in PBS (pH 7.4; Gibco; Thermo Fisher Scientific, Inc., Waltham, MA, USA) containing 1\% penicillin-streptomycin (Gibco; Thermo Fisher Scientific, Inc.). Subsequently, cartilage slices were minced with sterile ophthalmic scissors and transferred into digestion buffer containing $0.25 \%$ trypsin (Sigma-Aldrich, Merck KGaA, Darmstadt, Germany) for $30 \mathrm{~min}$ at $37^{\circ} \mathrm{C}$. The supernatant containing trypsin was discarded following centrifugation at $4^{\circ} \mathrm{C}$ for $10 \mathrm{~min}$ at $1,000 \mathrm{x}$ g and the trypsinized cartilage was digested again using $2 \mathrm{mg} / \mathrm{l}$ type IV collagenase (Sigma-Aldrich; Merck KGaA) for $4-6 \mathrm{~h}$ at $37^{\circ} \mathrm{C}$. Following the digestion, the cells were washed with Dulbecco's modified Eagle's medium (DMEM; Gibco; Thermo Fisher Scientific, Inc.) containing $10 \%$ fetal bovine serum (FBS; Gibco; Thermo Fisher Scientific, Inc.) and centrifuged at $800 \mathrm{x} \mathrm{g}$ for $10 \mathrm{~min}$ at $4^{\circ} \mathrm{C}$ prior to being cultured. The quantity of chondrocytes obtained was determined using a hemocytometer, and $0.4 \%$
Trypan blue dye (Sigma-Aldrich, Merck KGaA) was used to assess the viability of the cells. Chondrocytes were cultured in a $25 \mathrm{~cm}^{2}$ culture flask (Corning, Inc., Corning, NY, USA) in DMEM supplemented with $1 \%$ glutamine and $10 \%$ FBS at $37^{\circ} \mathrm{C}$ in $5 \% \mathrm{CO}_{2}$. Half of the medium was replaced once every 3 days with an equal quantity of fresh medium. Cells between passages 3 and 10 were used in the present study.

Normal chondrocytes were treated with $10 \mathrm{ng} / \mathrm{ml} \mathrm{IL-1 \beta}$ for 12,24 and $48 \mathrm{~h}$ for OA chondrocyte preparation. The cell treatment groups including the control group, IL-1 $\beta$ treatment group, IL-1 $\beta$ treatment with miR-98 inhibitor transfection group and corresponding control, miR-98 mimic transfection group and miR-98 mimic control group.

Transfections with miRNA mimics and inhibitors. In order to manipulate the cellular function of miR-98 in mouse chondrocytes, the present study used specific antisense oligonucleotides to miRNAs in order to inhibit miRNA function, and specific miRNA precursors to increase miRNA expression. Briefly, following culture of mouse chondrocytes to $90 \%$ confluence, they were transfected with 3-100 nM miR-98 mimic, miR-98 inhibitor or negative controls (Sangon Biotech Co., Ltd., Shanghai, China) using Lipofectamine 2000 (Invitrogen; Thermo Fisher Scientific, Inc.) according to the manufacturer's protocol. The expression levels of miR-98 were detected $24 \mathrm{~h}$ post-transfection in order to confirm the optimal concentration of miR-98 mimic, miR-98 inhibitor or negative controls had been used. The final concentration used in the present study was $50 \mathrm{nM}$.

RNA extraction and reverse transcription-quantitative polymerase chain reaction $(R T-q P C R)$. Total RNA was extracted using TRIzol reagent (Invitrogen; Thermo Fisher Scientific, Inc.) according to the manufacturer's protocol, and the concentration of total RNA was determined using NanoDrop 2000 (Thermo Fisher Scientific, Inc.). cDNA synthesis for the detection of target genes and miRNA was performed with $1 \mu \mathrm{g}$ total RNA using PrimeScript ${ }^{\mathrm{TM}} 1$ st Strand cDNA Synthesis kit (Takara Bio, Inc., Otsu, Japan) according to the manufacturer's protocol. The mRNA expression levels of Fas cell surface death receptor (Fas), caspase-3, caspase-8, Bcl-2-associated X protein (Bax), Bcl-2 and GAPDH, and the expression levels of miR-98 and U6 were analyzed using SYBR Green PCR Master Mix kit (Takara Bio, Inc.) according to the manufacturer's protocol. Primer sequences are presented in Table I. All oligonucleotide primers were synthesized by Sangon Biotech Co., Ltd. (Shanghai, China). qPCR reactions (denaturation: $95^{\circ} \mathrm{C}, 30 \mathrm{sec} 1$ cycle; PCR reaction: $95^{\circ} \mathrm{C}, 5 \mathrm{sec}, 60^{\circ} \mathrm{C}, 40 \mathrm{sec} 45$ cycles) were performed on an ABI7900 Real-Time system (Applied Biosystems; Thermo Fisher Scientific, Waltham, MA, USA). The Cq values were analyzed using the comparative $\mathrm{Cq}(\Delta \Delta \mathrm{Cq})$ method (18) and the quantity of target mRNA was obtained by normalizing to the endogenous references (GAPDH or U6), relative to the control. All experiments were repeated at least six times.

Western blotting. Whole cell lysates were obtained using RIPA Protein Extraction reagent (Beyotime Institute of Biotechnology, Inc., Haimen, China) plus EDTA-free 
Protease Inhibitor Cocktail tablets (Roche Diagnostics, Basel, Switzerland). Protein concentrations were detected using the BCA Protein Assay kit. Equal amounts of protein $(25 \mu \mathrm{g})$ were separated by $12.5 \%$ SDS-PAGE. Total proteins were electrotransferred onto polyvinylidene fluoride membranes (Merck KGaA) and blocked with 3\% bovine serum albumin (Beyotime Institute of Biotechnology, Inc., Haimen, China) in Tris-HCl buffered saline following electrophoresis. Bcl-2 (1,5071; $28 \mathrm{kD} ; 1: 2,000)$ and $\beta$-actin antibodies (12262; $42 \mathrm{kD} ; 1: 2,000)$ were purchased from Cell Signaling Technology, Inc. (Danvers, MA, USA). Following the primary antibody incubation at $4^{\circ} \mathrm{C}$ overnight, membranes were incubated with horseradish peroxidase-conjugated species-specific secondary antibody (sc-2031, 1:5,000; Santa Cruz Biotechnology, Inc.) at $37^{\circ} \mathrm{C}$ for $1 \mathrm{~h}$ and the proteins were visualized using an enhanced chemiluminescence kit (Pierce; Thermo Fisher Scientific, Inc.). The results of the western blotting were analyzed using Gel-Pro Analyzer version 4.0 (Media Cybernetics, Inc., Rockville, MD, USA). All experiments were repeated at least three times. Densitometric levels of target protein were semi-quantified and normalized to $\beta$-actin, relative to untransfected cells.

Terminal deoxynucleotidyl transferase dUTP nick end labeling (TUNEL) staining. TUNEL staining was performed $24 \mathrm{~h}$ post-transfection according to the manufacturer's protocol (Merck KGaA). The chondrocytes were fixed in cold $4 \%$ paraformaldehyde in PBS for $20 \mathrm{~min}$ at room temperature and washed three times in cold PBS. Subsequently equilibration buffer was added at room temperature for $10 \mathrm{~min}$. The fixed and permeabilized samples were incubated with DNaseI (300 U/ml in $50 \mathrm{mM}$ Tris- $\mathrm{HCl}, \mathrm{pH} 7.5$ ) for $10 \mathrm{~min}$ at room temperature in order to induce DNA strand breaks prior to labeling procedures. Reaction mix (10-50 $\mu 1$ well to immerse all samples) was added to the cells and incubated at $37^{\circ} \mathrm{C}$ for $2 \mathrm{~h}$ in a humidified atmosphere in the dark. Subsequently, the chondrocytes were rinsed with PBS three times for $5 \mathrm{~min}$. Hoechst 33342 solution was applied for $1 \mathrm{~min}$ in order to visualize all nuclei under a fluorescent microscope (Leica Microsystems GmbH, Wetzlar, Germany).

Cell proliferation assay. The cell proliferation rate was evaluated using an MTT proliferation assay. Briefly, following exposure to IL-1 $\beta$ (PHC0813; Thermo Fisher Scientific, Inc.) or transfection with recombinant plasmids, cells were cultured in 96-well plates and were washed twice with PBS at 24 and $48 \mathrm{~h}$. Subsequently, $0.5 \mathrm{mg} / \mathrm{ml}$ MTT was added to each well and the cells were incubated for $4 \mathrm{~h}$ at $37^{\circ} \mathrm{C}$ in $5 \% \mathrm{CO}_{2}$. The medium was replaced with $200 \mu \mathrm{l}$ dimethyl sulfoxide, the plates were agitated for $15 \mathrm{~min}$ at room temperature and the absorbance was determined at $490 \mathrm{~nm}$ using a microplate spectrophotometer.

Luciferase reporter constructs and luciferase assay. cDNA oligonucleotides (Sangon Biotech Shanghai, Co., Ltd., Shanghai, China) containing the putative miR-98 target site according to miRBase sequence database (http://www. mirbase.org) within the 3'-UTR of the target gene were synthesized with Hind III and Sac I restriction enzyme digestion (Takara Biotechnology Co., Ltd., Dalian, China) sites and cloned into the multiple cloning site of the pMIR-REPORT Luciferase vector (Ambion; Thermo Fisher Scientific, Inc.). An additional pMIR-REPORT Luciferase construct containing mutant 3'-UTR was generated as a control. Subsequently, HEK293 cells (Cell bank of Chinese Academy of Sciences, Shanghai, China) were cultured in 24-well plates with DMEM and 10\% FBS for $24 \mathrm{~h}$ and then were transfected with each reporter construct, alongside miR-98 mimic or miR-98 inhibitor, using Lipofectamine 2000 reagent (Invitrogen; Thermo Fisher Scientific, Inc.) according to the manufacturer's protocol. Firefly luciferase activity was quantified at $48 \mathrm{~h}$ post-transfection using the dual-luciferase reporter assay system (Thermo Fisher Scientific, Inc.).

Statistical analysis. Statistical analysis was conducted with SPSS 18.0 (SPSS, Inc., Chicago, IL, USA). All of the experiments were performed with samples in triplicate or greater. Data are presented as the mean \pm standard deviation of six independent experiments. One-way analysis of variance with Tukey HSD post hoc t tests were used to determine levels of statistical significance. Analysis of variance was used to determine statistical significance of the differences between the treatment groups. $\mathrm{P}<0.05$ was considered to indicate a statistically significant difference.

\section{Results}

Relative expression of miR-98 and apoptotic factors in chondrocytes following IL-1 $\beta$ treatment. In order to investigate whether the expression of miR-98 was induced by IL-1 $\beta$, mouse primary chondrocytes were incubated with IL-1 $\beta$ (10 $\mathrm{ng} / \mathrm{ml})$ for 12,24 and $48 \mathrm{~h}$. The expression levels of miR-98 were upregulated 2.75 -fold at $24 \mathrm{~h}$ and 3.61 -fold at $48 \mathrm{~h}$. The expression of miR-98 was significantly increased following incubation with $10 \mathrm{ng} / \mathrm{ml} \mathrm{IL-1} \beta$ for 24 and $48 \mathrm{~h}$ compared with in the $0 \mathrm{~h}$ treatment group $(\mathrm{P}<0.05$; Fig. $1 \mathrm{~A})$. The effects of IL-1 $\beta$ on apoptotic factors were also determined by examining their expression levels, including Fas, caspase-3, caspase-8, Bax and Bcl-2. Exposure of primary chondrocytes to IL-1 $\beta(10 \mathrm{ng} / \mathrm{ml})$ for $48 \mathrm{~h}$ significantly reduced the expression levels of Bcl-2 at the mRNA and protein level ( $\mathrm{P}<0.05$; Fig. 1A and $\mathrm{B})$. The mRNA expression levels of Fas, caspase-3, caspase- 8 and Bax were significantly greater following IL-1 $\beta$ stimulation for $48 \mathrm{~h}(\mathrm{P}<0.05$; Fig. 1A).

miR-98 affects apoptosis and proliferation of chondrocytes. In order to determine the effects of silencing miR-98 on mouse chondrocyte apoptosis and proliferation, miR-98 inhibitor was transfected into primary chondrocytes, and the chondrocytes were subsequently treated with $10 \mathrm{ng} / \mathrm{ml}$ IL-1 $\beta$ for 24 and $48 \mathrm{~h}$. A TUNEL assay was performed to detect the number of apoptotic cells. As presented in Fig. 2A, transfection with the miR-98 inhibitor reduced the number of TUNEL-positive cells compared with transfection with miR-98 inhibitor control or treatment withIL-1 $\beta$ only. Therefore, it is possible that downregulation of miR-98 expression in OA may be effective in the prevention of IL-1 $\beta$-induced chondrocyte apoptosis. An MTT assay was used to examine the proliferation rate of chondrocytes under 
Table I. Primer sequences for quantitative polymerase chain reaction.

\begin{tabular}{lll}
\hline Gene & \multicolumn{1}{c}{ Sense (5'-3') } & \multicolumn{1}{c}{ Antisense (5'-3') } \\
\hline miR-98 & ACACTCCAGCTGGGTGAGGTAGTAAGT & CTCAACTGGTGTCGTGGAGTC \\
U6 & CTCGCTTCGGCAGCACATTGC & AACGCTTCACGAATTTGCGT \\
Fas & AGACTGCGTGCCCTGCCAAGA & GGCCTGCCTGTTCAGTAACT \\
Caspase-3 & CTCGCTCTGGTACGGATGTG & TCCCATAAATGACCCCTTCATCA \\
Caspase-8 & CGTGCCTAATGGCGTTAACCA & AGCCTTGGCCAGCCGACCTT \\
Bax & GACCCGGTGCCTCAGGATGC & GTCTGTGTCCACGGCGGCAA \\
Bcl-2 & GCTACCGTCGTGACTTCGC & CCCCACCGAACTCAAAGAAGG \\
GAPDH & GACCCCTTCATTGACCTCAACTACA & GTCCACCACCCTGTTGCTGTAGCCA
\end{tabular}

miR-98, microRNA-98; Fas, Fas cell surface death receptor; Bcl-2, B-cell lymphoma 2; Bax, Bcl-2-associated X protein.
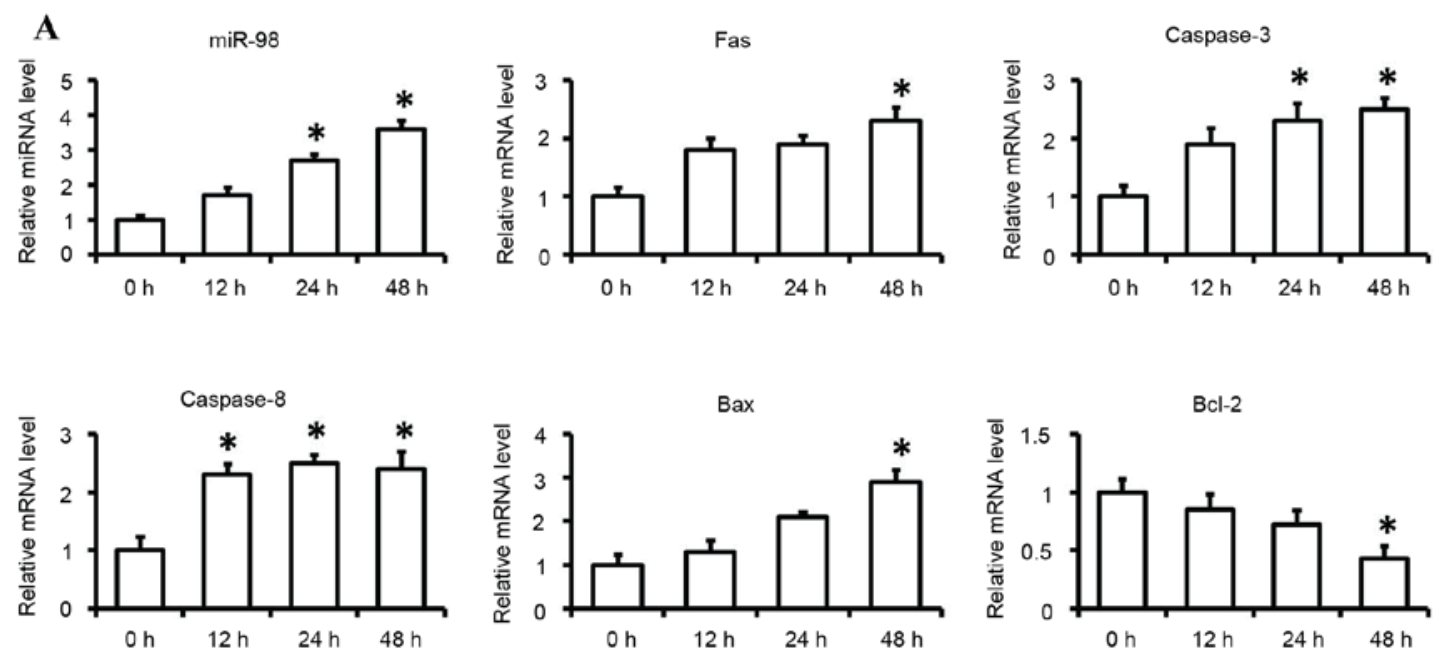

$\mathbf{B}$
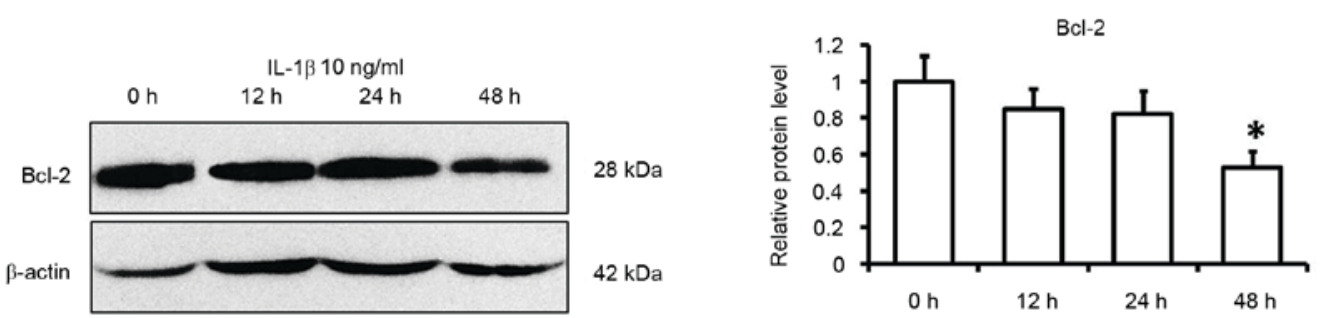

Figure 1. Expression levels of miR-98 and apoptotic factors in IL-1 $\beta$-induced chondrocytes. Cells were cultured with IL-1 $\beta$ (10 ng/ml) or control medium for 12, 24 and 48 h. (A) miR-98, Fas, caspase-3, caspase-8, Bax and Bcl-2 mRNA expression levels were determined by reverse transcription-quantitative polymerase chain reaction using U6 and GAPDH as internal controls. (B) Bcl-2 protein levels were determined by western blotting. Each data point was normalized to the control. Data are presented as the mean \pm standard error from six independent experiments. ${ }^{*} \mathrm{P}<0.05$ vs. control group $(0 \mathrm{~h})$. miR- 98 , microRNA- 98 ; IL-1 $\beta$, interleukin 1 $\beta$; Fas, Fas cell surface death receptor; Bcl-2, B-cell lymphoma 2; Bax, Bcl-2-associated X protein.

various conditions and it was determined that the rate of cell proliferation was significantly reduced following treatment withIL-1 $\beta$ at 24 and $48 \mathrm{~h}$ and this decrease was more pronounced after $48 \mathrm{~h}$ compared with at $24 \mathrm{~h}(\mathrm{P}<0.05$; Fig. $2 \mathrm{~B})$. However, transfection with the miR-98 inhibitor significantly alleviated the IL-1 $\beta$-induced decrease in cell proliferation at 48 h $(\mathrm{P}<0.05$; Fig. 2B).

miR-98 negatively regulates $B c l-2$ expression in mouse chondrocytes. The mRNA expression levels of miR-98 and apoptotic factors were compared following exposure to IL-1 $\beta$ in cultured chondrocytes using RT-qPCR, and an increase inmiR-98 expression was identified with a corresponding decrease in Bcl-2 expression following exposure to IL-1 $\beta$. In order to examine the effects of silencing miR-98 on Bcl-2 regulation, RT-qPCR and western blotting were performed following transfection of mouse chondrocytes with miR-98 inhibitor or mimic for $24 \mathrm{~h}$. RT-qPCR (Fig. 3A) and western blotting (Fig. 3B) revealed that the miR-98 mimic significantly reduced the mRNA and protein expression levels of Bcl-2 $(\mathrm{P}<0.05)$. Conversely, the miR-98 inhibitor significantly alleviated the IL- $1 \beta$-induced downregulation of Bcl-2 mRNA and protein expression ( $\mathrm{P}<0.05$; Fig. 3 ). These findings indicated that Bcl-2 may be a target gene of miR-98. 

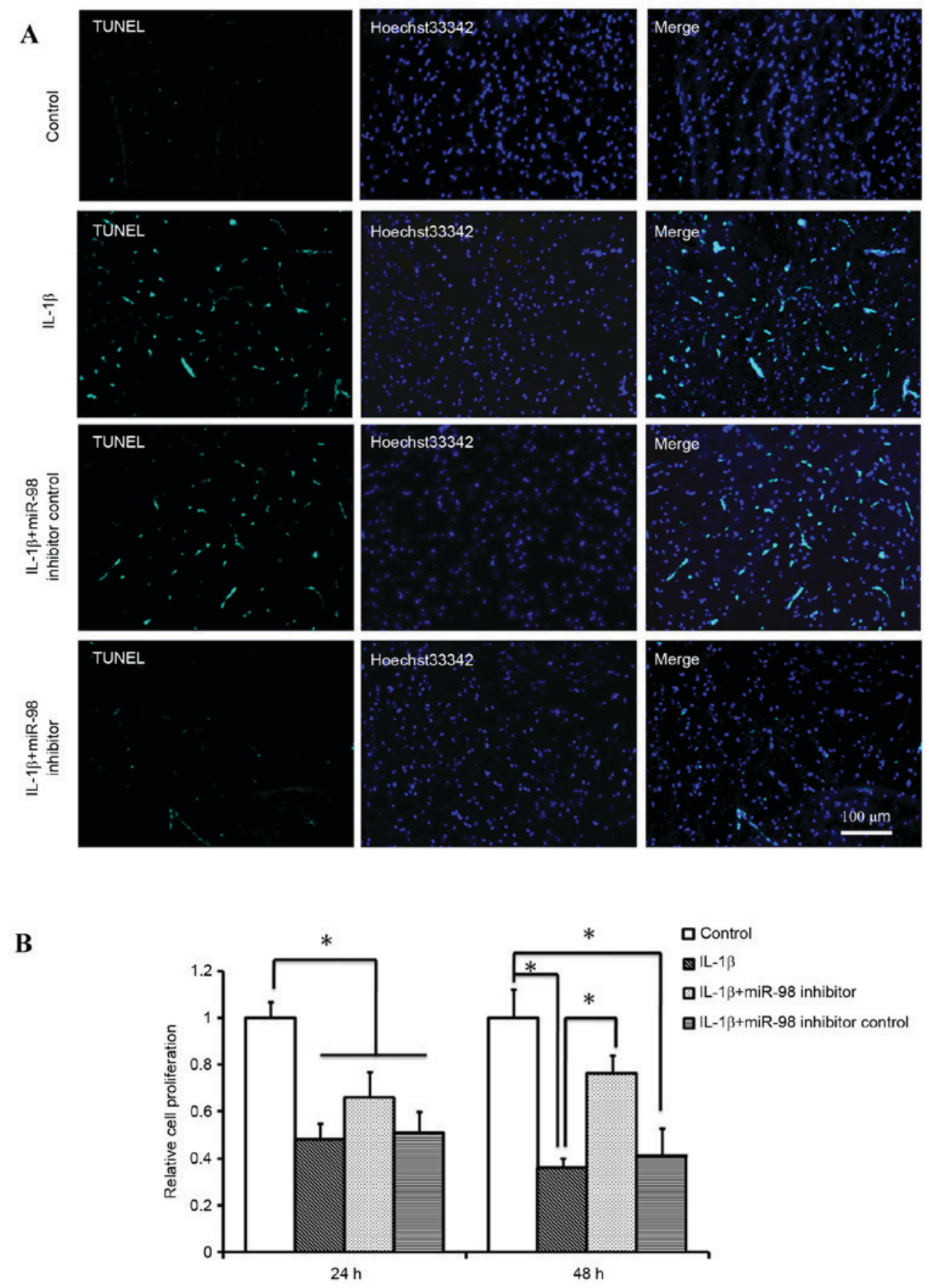

Figure 2. Effects of miR-98 on chondrocyte apoptosis and proliferation following IL-1 $\beta$ treatment. (A) Detection of apoptotic cells using TUNEL staining and (B) cell proliferation by MTT assay. Transfection with miR-98 inhibitor reduced the number of TUNEL-positive chondrocytes and promoted chondrocyte proliferation compared with transfection with miR-98 inhibitor control or treatment with IL-1 $\beta$ only (n=6/group). "P<0.05. IL-1 $\beta$, interleukin $1 \beta$; TUNEL, terminal deoxynucleotidyl transferase dUTP nick end labeling; miR-98, microRNA-98.

miR-98 targets the 3'-UTR of Bcl-2 and suppresses translation. The present study hypothesized that miR-98 may potentially target $\mathrm{Bcl}-2$, and the results revealed that transfection with a miR-98 inhibitor significantly increased the expression levels of Bcl-2 following treatment with IL-1 $\beta$. To validate the hypothesis that miR-98 regulates Bcl-2 expression following IL-1 $\beta$ stimulation, miR-98 mimic/mimic control were transfected into HEK293 cells. To confirm the efficiency of transfection, the expression levels of miR-98 were quantified using RT-qPCR. The mRNA and protein expression levels of Bcl-2 were also evaluated $24 \mathrm{~h}$ after $\mathrm{miR}-98 \mathrm{mimic} / \mathrm{mimic}$ control transfection. The relative expression levels of miR-98 were significantly increased following transfection of HEK293 cells with miR-98 mimic ( $\mathrm{P}<0.05$; Fig. 4A).RT-qPCR and western blot analysis revealed that the relative expression of Bcl-2 was significantly reduced in the miR-98 mimic transfection group compared with the mimic control group $(\mathrm{P}<0.05$; Fig. $4 \mathrm{~A})$. A dual luciferase reporter assay was performed to investigate whether miR-98 directly binds to the 3'-UTR of Bcl-2. It was determined that co-transfection with miR-98 mimic and pMIR-Bcl-2-wild type reporter plasmids significantly reduced the luciferase activity in HEK293 cells compared with in the 
$\mathbf{A}$

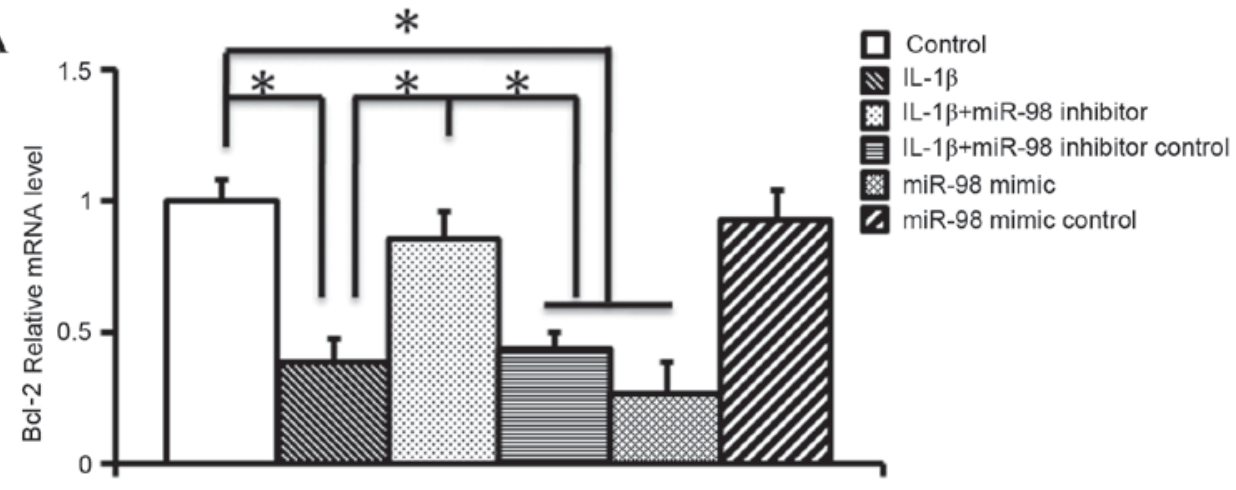

B
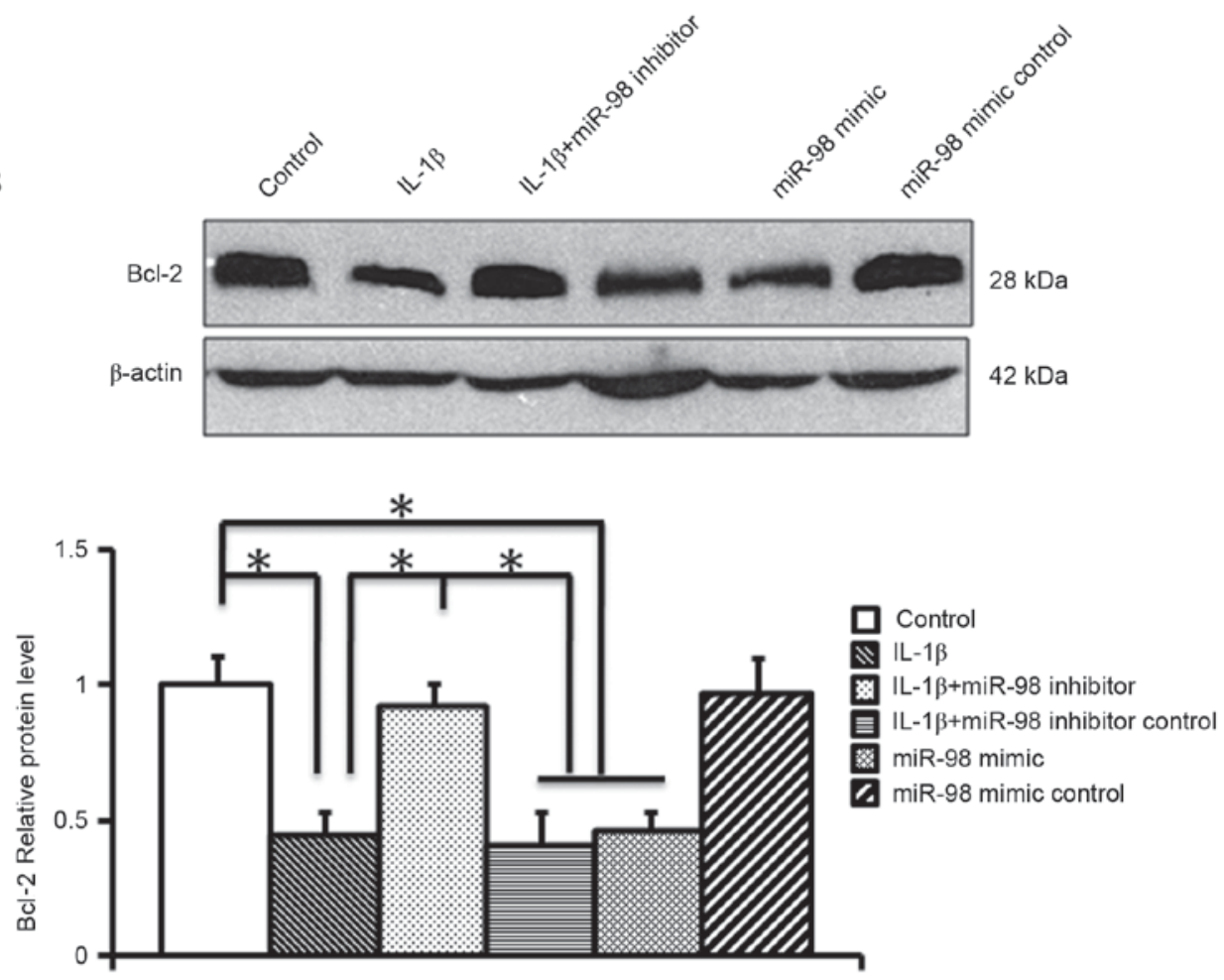

Figure 3. Analysis of the role of miR-98 in the expression of Bcl-2 in IL-1 $\beta$-treated chondrocytes. (A) Reverse transcription-quantitative polymerase chain reaction analysis of the mRNA expression of Bcl-2. (B) Western blot analysis of the protein levels of Bcl-2. miR-98 inhibitor significantly alleviated IL-1 $\beta$-induced downregulation of Bcl-2 at the mRNA and protein level ( $\mathrm{n}=6 /$ group). ${ }^{*} \mathrm{P}<0.05$. Bcl-2, B-cell lymphoma 2; IL-1 $\beta$, interleukin-1 $\beta$; miR-98, microRNA-98.

control group $(\mathrm{P}<0.05$; Fig. 4B). The results for the control luciferase assay was identical with the mimic control (data not shown).

\section{Discussion}

$\mathrm{OA}$ is the most prevalent arthropathy and frequently leads to chondrocyte apoptosis. Age, obesity, history of joint trauma, repeated injury, overuse and joint dysplasia are possibly involved in the etiology of OA, which may lead to serious joint pain, functional impairment or irreversible functional disability, along with reduced quality of life (19). The pathogenesis of OA is primarily associated with age and has been confirmed to increase with the median age of the population (20). Previous studies have indicated that numerous human diseases are associated with alterations in the expression of miRNAs, and several reports on miRNA profiling of human cartilage lesions have already been published $(5,21)$. Various algorithms may be used to predict potential mRNA targets; however, only a few miRNAs have been validated and assigned to specific mRNAs. To the best of our knowledge, the present study is the first to suggest that miR-98 serves an important role in chondrocyte apoptosis by regulating Bcl-2 expression in an IL-1 $\beta$ conditional cultured system in vitro. Previous studies identified various miRNAs that may be involved in chondrogenesis and OA $(14,22)$. The role of miR-98 in tumor apoptosis remains to be elucidated. Previous studies have reported that miR-98 expression is associated with tumor cell growth $(15,23)$. Clinical studies have suggested that miR-98 expression is associated with head and neck cancer development (24) and may be downregulated in nasopharyngeal carcinoma (25). In addition, microarrays and qPCR have confirmed that miR-98 is upregulated in primary breast cancer specimens (26). However, contradictory findings have been reported in two other recent 
A
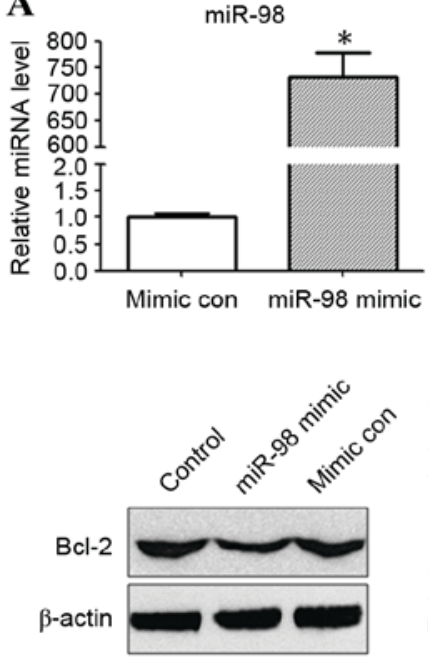

$\mathrm{Bcl}-2$
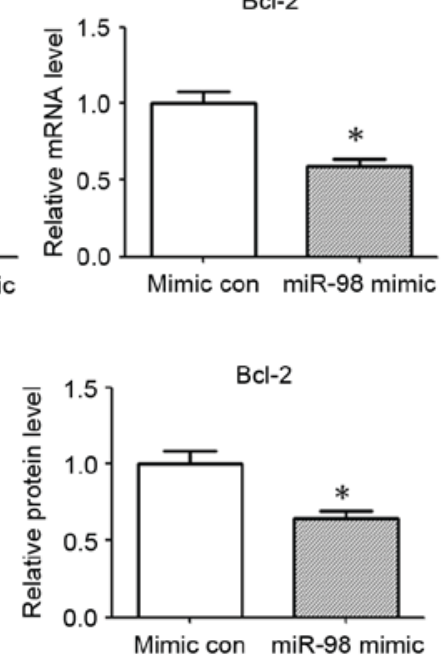

B
Bcl-2 3' UTR 5' UCGAAUCAGCUAUUUACUGCCAAA 3' III | | IIII| II |

miR-98 mmu 3' UGUUAUAUGUUGAAUGAUGGAGU 5'

Figure 4. Bcl-2 was identified as a target gene of miR-98. Cells were transfected with $50 \mathrm{nM}$ miR-98 mimic or control mimic for 24 h. (A) Reverse transcription-quantitative polymerase chain and western blotting were performed to evaluate the expression levels of miR-98 and Bcl-2. (B) Sequence alignment (miRBase sequence database; http://www.mirbase.org) of miR-98 with 3'-UTR of Bcl-2. Luciferase reporter activity was significantly decreased following transfection with the miR-98 mimic compared with in the control group (lower panel) (n=6/group). * $\mathrm{P}<0.05$ vs. control group. miR-98, microRNA-98; Bcl-2, B-cell lymphoma 2; 3'-UTR, 3'-untranslated region; con, control.

studies. Chen et al (27) demonstrated that miR-25 was upregulated in OA chondrocytes, whereas Miyaki et al (28) stated that this miRNA was downregulated in OA cartilage. Using an in vitro model of mouse OA chondrocytes, the present study detected the expression levels of miR-98 using RT-qPCR; the results demonstrated that the expression levels of miR-98 were increased in OA chondrocytes following in vitro treatment with IL-1 $\beta$, as previously reported (11). The expression levels of apoptotic factors following IL-1 $\beta$ stimulation were also detected in order to determine the effects of miR-98 on the apoptosis of mouse chondrocytes.

Removal of undesirable cells is a biological process that functions to maintain homeostasis, and is termed apoptosis or programmed cell death (29). Apoptosis is controlled by two primary molecular signaling pathways: The extrinsic and intrinsic pathways $(30,31)$. The extrinsic apoptotic pathway refers to Fas ligand (FasL) binding to Fas receptor $(32,33)$. This forms the death-inducing signaling complex, which contains the Fas-associated death domain, caspase- 8 and caspase- 10 . Subsequently, cleavage and activation of executive caspase- 3 is induced by cleaved caspase-8; activated capase-3 consequently cleaves DNA molecules, leading to apoptosis $(34,35)$. It has previously been demonstrated that the Fas/FasL pathway exerts a principle role in the induction of apoptosis (36), and in many tumor and inflammation related diseases an alteration of the Fas/FasL pathway has been observed. The Bcl-2 protein family, which includes anti-apoptotic proteins, such as Bcl-2, Bcl-extra large and myeloid cell leukemia 1, and pro-apoptotic members, including Bcl-2 antagonist/killer 1 (Bak), Bax and Bcl-2-like protein 11, are important mediators of the intrinsic pathway. Overexpression of any of the Bcl-2-like pro-survival members, or the loss of the multi-Bcl-2-homology domain proteins Bak and Bax, may inhibit apoptosis through the intrinsic pathway (37). The present study used RT-qPCR to identify the mRNA expression levels of apoptotic factors. Fas, caspase-3, caspase- 8 and Bax expression levels were all increased following IL-1 $\beta$ stimulation; however, a decrease in Bcl-2 and a corresponding increase in miR-98 expression was detected following IL-1 $\beta$ exposure. Western blot analysis confirmed that $\mathrm{Bcl}-2$ protein expression levels were significantly reduced. In order to determine whether reduced Bcl-2 expression was regulated by the upregulation of miR-98 RT-qPCR and western blotting were performed, and revealed that IL-1 $\beta$ treatment reduced Bcl-2 expression via post-transcriptional gene regulation involving the activation of miR-98. A dual luciferase reporter assay confirmed that miR-98 may target the 3'-UTR of Bcl-2, which leads to translational suppression.

Chondrocytes are an important component of arthrosis, which serve a role in maintaining normal cartilage homeostasis and structural integrity, and account for only $5 \%$ of the total cartilage volume; production of the extracellular matrix and its enzymatic degradation are maintained by chondrocytes (38). The tilting of this balance in favor of catabolic events may lead to the loss of articular cartilage, as observed in OA. Therefore, maintaining the integrity of the articular cartilage is the primary function of chondrocytes. In addition, chondrocyte damage may lead to matrix degeneration, and may be associated with the onset and progression of OA $(39,40)$. Previous studies have indicated that chondrocyte apoptosis may be associated with OA pathogenesis, and studies performed in situ have identified a higher number of apoptotic chondrocytes in OA compared with in normal samples $(41,42)$. Therefore, the effective prevention of chondrocyte apoptosis may be a potential therapeutic strategy for the treatment of OA. In the present study, miR-98 inhibited the upregulation of Bcl-2 expression, which may be one of the primary causes of articular chondrocyte apoptosis. However, the function of miR-98 in chondrocyte apoptosis via the inhibition of its target genes remains to be fully elucidated, as computational analyses frequently state that one miRNA may have hundreds of target genes (43). Therefore, chondrocyte apoptosis may 
be involved in numerous molecular networks associated with apoptosis and growth.

In conclusion, the present study demonstrated that the expression of miR-98 was induced by IL-1 $\beta$ treatment in mouse primary chondrocytes and that a miR-98 inhibitor may prevent the downregulation of Bcl-2 induced by IL-1 $\beta$ in chondrocytes. These results suggested that silencing miRNA-98 may prevent chondrocyte apoptosis.

\section{Acknowledgements}

The authors would like to thank Professor Jiesheng Gao (The Second Xiangya Hospital of Central South University, Changsha, China) for his guidance. The present study was supported by the National Natural Science Foundation of China (grant no. 81260286).

\section{References}

1. Aigner T, Söder S, Gebhard PM, McAlinden A and Haag J: Mechanisms of disease: Role of chondrocytes in the pathogenesis of osteoarthritis-structure, chaos and senescence. Nat Clin Pract Rheumatol 3: 391-399, 2007.

2. Li YH, Tavallaee G, Tokar T, Nakamura A, Sundararajan K, Weston A, Sharma A, Mahomed NN, Gandhi R, Jurisica I and Kapoor M: Identification of synovial fluid microRNA signature in knee osteoarthritis: Differentiating early- and late-stage knee osteoarthritis. Osteoarthritis Cartilage 24: 1577-1586, 2016.

3. Chen CZ, Li L, Lodish HF and Bartel DP: MicroRNAs modulate hematopoietic lineage differentiation. Science 303: 83-86, 2004.

4. Chen CZ and Lodish HF: MicroRNAs as regulators of mammalian hematopoiesis. Semin Immunol 17: 155-165, 2005.

5. Iliopoulos D, Malizos KN, Oikonomou P and Tsezou A: Integrative microRNA and proteomic approaches identify novel osteoarthritis genes and their collaborative metabolic and inflammatory networks. PLoS One 3: e3740, 2008.

6. Diaz-Prado S, Cicione C, Muiños-Lopez E, Hermida-Gómez T, Oreiro N, Fernández-López C and Blanco FJ: Characterization of microRNA expression profiles in normal and osteoarthritic human chondrocytes. BMC Musculoskelet Disord 13: 144, 2012.

7. Goldring MB and Marcu KB: Cartilage homeostasis in health and rheumatic diseases. Arthritis Res Ther 11: 224, 2009.

8. Mayan MD, Gago-Fuentes R, Carpintero-Fernandez P, Fernandez-Puente P, Filgueira-Fernandez P, Goyanes N, Valiunas V, Brink PR, Goldberg GS and Blanco FJ: Articular chondrocyte network mediated by gap junctions: Role in metabolic cartilage homeostasis. Ann Rheum Dis 74: 275-284, 2015.

9. Daheshia M and Yao JQ: The interleukin 1beta pathway in the pathogenesis of osteoarthritis. J Rheumatol 35: 2306-2312, 2008.

10. Kapoor M, Martel-Pelletier J, Lajeunesse D, Pelletier JP and Fahmi H: Role of proinflammatory cytokines in the pathophysiology of osteoarthritis. Nat Rev Rheumatol 7: 33-42,2011.

11. Jones SW, Watkins G, Le Good N, Roberts S, Murphy CL, Brockbank SM, Needham MR, Read SJ and Newham P: The identification of differentially expressed microRNA in osteoarthritic tissue that modulate the production of TNF-alpha and MMP13. Osteoarthritis Cartilage 17: 464-472, 2009.

12. Miyaki S, Nakasa T, Otsuki S, Grogan SP, Higashiyama R, Inoue A, Kato Y, Sato T, Lotz MK and Asahara H: MicroRNA-140 is expressed in differentiated human articular chondrocytes and modulates interleukin-1 responses. Arthritis Rheum 60: 2723-2730, 2009.

13. Yamasaki K, Nakasa T, Miyaki S, Ishikawa M, Deie M, Adachi N, Yasunaga Y, Asahara H and Ochi M: Expression of MicroRNA-146a in osteoarthritis cartilage. Arthritis Rheum 60: 1035-1041, 2009.

14. Park SJ, Cheon EJ and Kim HA: MicroRNA-558 regulates the expression of cyclooxygenase- 2 and IL-1 $\beta$-induced catabolic effects in human articular chondrocytes. Osteoarthritis Cartilage 21: 981-989, 2013.
15. Siragam V, Rutnam ZJ, Yang W, Fang L, Luo L, Yang X, Li M, Deng Z, Qian J, Peng C and Yang BB: MicroRNA miR-98 inhibits tumor angiogenesis and invasion by targeting activin receptor-like kinase-4 and matrix metalloproteinase-11. Oncotarget 3: 1370-1385, 2012

16. Du L, Schageman JJ, Subauste MC, Saber B, Hammond SM, Prudkin L, Wistuba II,Ji L, Roth JA, Minna JD and Pertsemlidis A: miR-93, miR-98, and miR-197 regulate expression of tumor suppressor gene FUS1. Mol Cancer Res 7: 1234-1243, 2009.

17. Li F, Li XJ, Qiao L, Shi F, Liu W, Li Y, Dang YP, Gu WJ, Wang XG and Liu W: miR-98 suppresses melanoma metastasis through a negative feedback loop with its target gene IL-6. Exp Mol Med 46: e116, 2014.

18. Livak KJ and Schmittgen TD: Analysis of relative gene expression data using real-time quantitative PCR and the 2(-Delta Delta C(T)) Method. Methods 25: 402-408, 2001.

19. Scharstuhl A, Schewe B, Benz K, Gaissmaier C, Bühring HJ and Stoop R: Chondrogenic potential of human adult mesenchymal stem cells is independent of age or osteoarthritis etiology. Stem Cells 25: 3244-3251, 2007.

20. Hung VW, Zhu TY, Cheung WH, Fong TN, Yu FW, Hung LK, Leung KS, Cheng JC, Lam TP and Qin L: Age-related differences in volumetric bone mineral density, microarchitecture, and bone strength of distal radius and tibia in Chinese women: A high-resolution pQCT reference database study. Osteoporos Int 26: 1691-1703, 2015.

21. Betel D, Wilson M, Gabow A, Marks DS and Sander C: The microRNA.org resource: Targets and expression. Nucleic Acids Res 36 (Database issue): D149-D153, 2008.

22. Lin EA, Kong L, Bai XH, Luan Y and Liu CJ: miR-199a, a bone morphogenic protein 2-responsive MicroRNA, regulates chondrogenesis via direct targeting to Smad1. J Biol Chem 284: 11326-11335, 2009.

23. Wendler A, Keller D, Albrecht C, Peluso JJ and Wehling M: Involvement of let-7/miR-98 microRNAs in the regulation of progesterone receptor membrane component 1 expression in ovarian cancer cells. Oncol Rep 25: 273-279, 2011.

24. Hebert C, Norris K, Scheper MA, Nikitakis N and Sauk JJ: High mobility group A2 is a target for miRNA-98 in head and neck squamous cell carcinoma. Mol Cancer 6: 5, 2007.

25. Alajez NM, Shi W, Hui AB, Bruce J, Lenarduzzi M, Ito E, Yue S, O'Sullivan B and Liu FF: Enhancer of Zeste homolog 2 (EZH2) is overexpressed in recurrent nasopharyngeal carcinoma and is regulated by miR-26a, miR-101, and miR-98. Cell Death Dis 1: e85, 2010.

26. Yan LX, Huang XF, Shao Q, Huang MY, Deng L, Wu QL, Zeng YX and Shao JY: MicroRNA miR-21 overexpression in human breast cancer is associated with advanced clinical stage, lymph node metastasis and patient poor prognosis. RNA 14: 2348-2360, 2008.

27. Chen CG, Thuillier D, Chin EN and Alliston T: Chondrocyte-intrinsic Smad3 represses Runx2-inducible matrix metalloproteinase 13 expression to maintain articular cartilage and prevent osteoarthritis. Arthritis Rheum 64: 3278-3289, 2012.

28. Miyaki S and Asahara H: Macro view of microRNA function in osteoarthritis. Nat Rev Rheumatol 8: 543-552, 2012.

29. Jin Z and El-Deiry WS: Overview of cell death signaling pathways. Cancer Biol Ther 4: 139-163, 2005.

30. Carrington PE, Sandu C, Wei Y, Hill JM, Morisawa G, Huang T, Gavathiotis E, Wei Y and Werner MH: The structure of FADD and its mode of interaction with procaspase-8. Mol Cell 22: 599-610, 2006

31. Tibbetts MD, Zheng L and Lenardo MJ: The death effector domain protein family: Regulators of cellular homeostasis. Nat Immunol 4: 404-409, 2003.

32. Barnhart BC, Lee JC, Alappat EC and Peter ME: The death effector domain protein family. Oncogene 22: 8634-8644, 2003.

33. Harper N, Hughes M, MacFarlane $M$ and Cohen GM: Fas-associated death domain protein and caspase- 8 are not recruited to the tumor necrosis factor receptor 1 signaling complex during tumor necrosis factor-induced apoptosis. J Biol Chem 278: 25534-25541, 2003.

34. Houston A and O'Connell J: The Fas signalling pathway and its role in the pathogenesis of cancer. Curr Opin Pharmacol 4: 321-326, 2004.

35. Thomas LR, Johnson RL, Reed JC and Thorburn A: The C-terminal tails of tumor necrosis factor-related apoptosis-inducing ligand (TRAIL) and Fas receptors have opposing functions in Fas-associated death domain (FADD) recruitment and can regulate agonist-specific mechanisms of receptor activation. J Biol Chem 279: 52479-52486, 2004. 
36. Kunes P, Krejsek J, Brtko M, Mandak J, Kolackova M, Trojackova Kudlova M and Andrys C: Neutrophil apoptosis by Fas/FasL: Harmful or advantageous in cardiac surgery? Thorac Cardiovase Surg 57: 1-6, 2009.

37. Borner C: The Bcl-2 protein family: Sensors and checkpoints for life-or-death decisions. Mol Immunol 39: 615-647, 2003.

38. Findlay DM and Atkins GJ: Osteoblast-chondrocyte interactions in osteoarthritis. Curr Osteoporos Rep 12: 127-134, 2014.

39. Hayes AJ, MacPherson S, Morrison H, Dowthwaite G and Archer CW: The development of articular cartilage: Evidence for an appositional growth mechanism. Anat Embryol (Berl) 203: 469-479, 2001

40. Pacifici M, Koyama E, Iwamoto M and Gentili C: Development of articular cartilage: What do we know about it and how may it occur? Connect Tissue Res 41: 175-184, 2000.
41. Hashimoto S, Ochs RL, Rosen F, Quach J, McCabe G, Solan J, Seegmiller JE, Terkeltaub R and Lotz M: Chondrocyte-derived apoptotic bodies and calcification of articular cartilage. Proc Natl Acad Sci USA 95: 3094-3099, 1998.

42. Kim HA and Blanco FJ: Cell death and apoptosis in osteoarthritic cartilage. Curr Drug Targets 8: 333-345, 2007.

43. Krek A, Grun D, Poy MN, Wolf R, Rosenberg L, Epstein EJ, MacMenamin P, da Piedade I, Gunsalus KC, Stoffel M and Rajewsky N: Combinatorial microRNA target predictions. Nat Genet 37: 495-500, 2005. 